\title{
Estudio exploratorio de los rasgos y facetas de la personalidad en estudiantes universitarios que realizan binge drinking
}

\section{Exploratory study of personality traits and facets in university students binge drinking}

Fecha de recepción: 04-01-2019

Fecha de aceptación: 24-03-2019

\author{
Patricia Motos Sellés \\ Universidad Internacional de Valencia \\ Área de Educación \\ María Teresa Cortés Tomás \\ Universitat de València \\ Departamento de Psicología Básica \\ José Antonio Giménez Costa \\ Universitat de València \\ Departamento de Psicología Básica \\ Beatriz Martín Del Río \\ Universidad Miguel Hernández
}

\section{resumen/abstract:}

Existen factores de personalidad que se encuentran relacionados con la práctica del binge drinking (BD) entre la población universitaria española, patrón de consumo asociado a graves implicaciones biopsicosociales. En este estudio se pretende explorar la relación que existe entre las dimensiones y facetas de la personalidad en función del nivel de gravedad del BD. Los participantes fueron 601 estudiantes de la Universidad Miguel Hernández de Elche comprendidos entre 18 y 20 años (64.1\% mujeres) cumplimentaron un autorregistro del patrón de consumo y la adaptación española del NEO-PI-R. Los grupos de hombres y mujeres BD se clasificaron según la intensidad y frecuencia del consumo. Los resultados muestran la existencia de ciertas relaciones entre algunas facetas de personalidad y los diferentes tipos de consumidores $\mathrm{BD}$. Concretamente, en los varones que realizan BD con mayor regularidad se recomienda atender concretamente a las facetas de las dimensiones de Apertura y Amabilidad. En cuanto a las mujeres BD más extremas, la dimensión de Neuroticismo es la que más destaca, concretamente la Impulsividad. Por último, sería importante trabajar aspectos relacionados con la faceta de Búsqueda de Sensaciones en los varones que consumen mayores cantidades.

There are personality factors that are related to the practice of binge drinking (BD) among Spanish university students, a pattern of consumption associated with serious biopsychosocial implications. The aim is to explore the relationship of the personality traits and facets according to the level of severity of BD. The participants were 601 students from the Miguel Hernández University of Elche between 18 and 20 years old (64.1\% women) completed a self-registration of the consumption pattern and the Spanish adaptation of the NEO-PI-R. BD groups have been differentiated by different intensity and frequency levels, both in males and females. The results show the existence of certain relationships between some facets of personality and the different types of BD consumers. Specifically, in men who perform BD more regularly, it is recommended to specifically attend facets of the Openness and Agreeableness dimensions. As for the most extreme $B D$ women, the dimension of Neuroticism is the one that stands out, specifically the Impulsivity. Finally, it would be important to work on aspects related to the Excitement Seeking among men who consume larger amounts. 


\section{palabras clave/keywords:}

Consumo intensivo de alcohol, jóvenes, personalidad.

Heavy drinking, young people, personality.

\section{Introducción}

Son numerosos los estudios con población universitaria que afirman que existen factores de la personalidad que se relacionan con la práctica del binge drinking -BD- (Adan, Forero y Navarro, 2017). Se trata de un patrón de consumo de tipo intermitente caracterizado por la realización de ingestas elevadas de alcohol en cortos periodos de tiempo -horas- (Cortés y Motos, 2015). Este tipo de conducta representa un problema de salud pública (Marczinski, Grant y Grant, 2009; SAMHSA, 2009) debido a las implicaciones biopsicosociales con las que está asociado (Cortés et al., 2012; ESPAD, 2016).

Este colectivo se caracteriza por una elevada Impulsividad (Bravo et al., 2018; Motos, Cortés, Giménez y Cadaveira, 2015) y Búsqueda de Sensaciones (Adan et al., 2017; Lac y Donaldson, 2016; Mushquash, Stewart, Mushquash, Comeau y McGrath, 2014). No obstante, es importante atender a otros rasgos de personalidad que también están influyendo en la conducta de consumo de esta población, como son las puntuaciones elevadas en Extraversión y Neuroticismo, o las bajas en Amabilidad y Responsabilidad (Erevik, Pallesen, Vedaa, Yreassen y Torsheim, 2017; Hakulinen y Jokela, 2018; Lac y Donaldson, 2016; Motos et al., 2015).

Un aspecto controvertido es el relacionado con el rasgo de Apertura, ya que algunos autores como Mezquita, Stewart y Ruipérez (2010) o Ruiz, Pincus y Dickinson (2003) lo definieron como poco representativo, mientras que Martin, Groth, Longo, Rocha y Martens (2015) encontraron puntuaciones elevadas en las mujeres BD.

Además, hay que matizar que la mayoría de los estudios únicamente atienden a las dimensiones de la personalidad de orden superior, ignorando el papel que otras facetas de orden inferior podrían ejercer en la identificación de patrones de consumo de alcohol y trastornos asociados (Ruiz et al., 2003).

Ruiz et al. (2003) concluyeron que un mayor consumo de alcohol se relacionaba con altos niveles en las facetas de Impulsividad y de Búsqueda de Sensaciones, junto con bajos niveles en las facetas de Franqueza, Altruismo, Sensibilidad a los Demás, Competencia, Sentido del Deber, Necesidad de Logro, Autodisciplina y Reflexión.

Por otra parte, en adolescentes consumidores de alcohol durante el fin de semana, Ibánez et al. (2015) encontraron puntuaciones elevadas en las facetas de Gregarismo y Búsqueda de Sensaciones y niveles más bajos en Reflexión-Deliberación.

Recientes estudios centrados en el análisis de las diferencias entre hombres y mujeres con respecto a la personalidad, destacan que las mujeres $\mathrm{BD}$ muestran mayores niveles en las facetas de Ansiedad y Depresión (Adan et al., 2017; Lac y Donaldson, 2016), mientras que 
los varones presentan una personalidad más impulsiva y una necesidad de buscar nuevas experiencias (Adan et al., 2017; Lang et al., 2012; Motos et al., 2015).

Las últimas investigaciones recogidas en la revisión de Adan et al. (2017) han demostrado la existencia de diferentes subtipos de binge drinkers según los rasgos de personalidad que los definen. Concretamente, Lannoy, Billieux, Poncin, y Maurage (2017), señalan la existencia de tres tipos de bebedores universitarios BD en función de la personalidad: el emocional que se define por mostrar mayor interés por buscar nuevas sensaciones y Urgencia, el recreativo que se caracteriza por una falta de Premeditación y Perseverancia, y el peligroso, cuyo rasgo de personalidad principal es la Impulsividad.

Por su parte, Zhang, Bray, Zhang y Lanza (2015), han encontrado dos perfiles de consumidores según la frecuencia de BD: uno con una frecuencia más moderada, con puntuaciones elevadas en Responsabilidad y bajas en Extraversión, Apertura y Amabilidad, y un segundo perfil con una frecuencia más intensa, en el que predominan una elevada Extraversión, Apertura y Amabilidad.

Disponer de este tipo de información más precisa, considerando la gravedad del BD y la diferenciación por sexos, permitirá concretar la intervención a realizar sobre cada uno de estos grupos.

Al mismo tiempo, no puede obviarse que se utilizan diversas definiciones de BD, no comparables entre sí (Cortés, Giménez, Motos y Sancerni, 2016).

La revisión más reciente sobre la operacionalización de este patrón de consumo (Cortés y Motos, 2015; Cortés, Giménez, Motos y Sancerni, 2017a) recomienda el consumo de 6 o más UBEs (Unidades de Bebida Estándar) españolas en mujeres y 7 o más en hombres, durante un intervalo de 2 horas, al menos una vez en los últimos seis meses.

Con el fin de avanzar en la comprensión del papel que juega la personalidad en la heterogeneidad del patrón BD en universitarios, tratando de verificar los resultados poco precisos obtenidos hasta el momento, se explorará la relación de las dimensiones y facetas de la personalidad en los diferentes tipos de consumidores BD y NoBD establecidos. Para ello, se recurrirá al NEO-PI-R, al ser un instrumento que incluye tanto las dimensiones como las facetas de la personalidad.

A partir de los resultados de la investigación precedente, se espera que los jóvenes BD puntúen más en las dimensiones y facetas de personalidad relacionadas con Neuroticismo y Extraversión, y menos en Amabilidad y Responsabilidad. En cuanto al sexo, las mujeres BD mostrarán mayores niveles en las facetas de Ansiedad y Depresión, mientras que los varones BD lo harán en Impulsividad y Búsqueda de Sensaciones. Además, es probable que se detecten diferencias en los rasgos de personalidad en función de la intensidad del consumo.

\section{Método}

\section{Participantes}

Participaron 601 estudiantes voluntarios de la Universidad Miguel Hernández de Elche (64.1\% mujeres; $35.9 \%$ varones), entre 18 y 20 años de edad ( Md =19.25 años; DT = .795). 
De los encuestados, el 63.9\% (384) cumplió con los criterios de BD (ingesta de 6 o más UBEs en mujeres y 7 o más en hombres, durante 2 horas, al menos una vez en los últimos seis meses), 129 varones $(33.6 \%$,) y 255 mujeres $(66.4 \%)$. Entre los estudiantes que no realizaron BD, 87 eran varones $(40.1 \%)$ y 130 eran mujeres (59.9\%). Estos jóvenes consumidores se identificaron como NoBD por no alcanzar los límites de consumo para realizar un $\mathrm{BD}$, según la definición anteriormente descrita.

\section{Variables e instrumentos}

Patrón de consumo: Las variables asociadas con el consumo de alcohol (número y tipo de bebidas y momento en que se produjo la ingesta) se recogieron mediante un autoinforme. Las cantidades de consumo se convirtieron a Unidades de Bebida Estándar (UBEs), siguiendo la definición de una UBE española -1 consumición de una bebida destilada $=2$ UBEs; 1 bebida fermentada = 1 UBE- (Rodríguez-Martos, Gual y Llopis, 1999). La suma de las UBEs consumidas en cada episodio de consumo permitió identificar el momento de mayor consumo por cada estudiante (mayor número de UBEs consumidas en una sesión $B D$ -2 horas-).

La frecuencia de BD se obtuvo a partir de la contabilización de días en los que se había consumido de esta manera durante el último medio año (número total de días de $B D$ en los últimos seis meses). A partir de estas dos variables, se pudieron identificar los estudiantes que realizaron $\mathrm{BD}$.

Personalidad: Se utilizó la adaptación española del NEO-PI-R (Costa y McCrae, 1999) compuesto por 240 ítems, con formato de respuesta Likert de 5 opciones. En éste se diferencian cinco dimensiones: Neuroticismo (N), Extraversión (E), Apertura (O), Afabilidad (A) y Responsabilidad (C) y un total de 30 facetas (6 para cada dimensión). El instrumento presenta una buena consistencia interna con población universitaria BD -alpha de Cronbach entre .71 y .85- (Mezquita et al., 2010; Ruiz et al., 2003). En este estudio, se alcanza el .74.

\section{Procedimiento}

La información se recogió mediante una batería de instrumentos que los estudiantes de primer curso de la Universidad Miguel Hernández (Elche) autocumplimentaron durante el curso académico 2016. La batería se presentó como una práctica voluntaria dentro de la asignatura de documentación. A los estudiantes se les explicó en las aulas la investigación, la finalidad general de la misma y se presentaron los instrumentos incluidos en la recogida de información. La investigación se llevó a cabo conjuntamente con la Universitat de València. Todos aquellos que decidieron participar en esta práctica se comprometieron a recoger un máximo de 5 pruebas, invitando a conocidos consumidores de alcohol con edades comprendidas entre los 18 y 20 años a cumplimentar la batería de instrumentos. Todos los entrevistados, tanto los estudiantes de primer curso como los invitados por los mismos, realizaron la entrevista de manera personal en despachos de la universidad, contando con la presencia de la investigadora coordinadora del pase en esta universidad. Los voluntarios firmaron una hoja de consentimiento tras ser informados del objetivo de la investigación y previo al inicio de la cumplimentación de los pases. 


\section{Diseño}

El diseño de investigación es cuasiexperimental de corte trasversal. Se evaluaron diferentes dimensiones de la personalidad en función del sexo y del tipo de consumo de alcohol que realizan actualmente los jóvenes.

\section{Análisis de datos}

Se realizaron análisis descriptivos para obtener información sociodemográfica.

Los grupos de consumidores de BD se establecieron a partir de dos análisis de conglomerados de dos fases, uno para cada sexo. Se utilizó este procedimiento de extracción, ya que selecciona automáticamente el número óptimo final de grupos (conglomerados) según las variables de interés. En este caso, para identificar a los diferentes tipos de consumidores $\mathrm{BD}$ se utilizaron las variables que definen el propio BD: mayor número de UBEs consumidas en una sesión $B D$ y número total de días de BD en los últimos seis meses. El análisis de conglomerados extrajo un total de tres grupos de consumidores BD en cada sexo.

También se efectuaron análisis de varianza (ANOVA) para comprobar la existencia de diferencias significativas entre los grupos BD, así como también entre los NoBD, diferenciados por sexos. Se recurrió a las pruebas a posteriori (Tukey/Games-Howell) para mostrar donde se encontraban las diferencias.

Todos los análisis fueron realizados con el paquete estadístico IBM SPSS-22.

\section{Resultados}

\section{Conglomerados según la definición del BD}

Los resultados de los dos análisis de conglomerados pueden observarse en la tabla 1. Tal como se ha indicado en el apartado anterior, para cada sexo se diferenciaron tres grupos de BD. En los varones: BDV1 (58.91\% de la muestra), BDV2 (13.95\%) y BDV3 (27.13\%); y en las mujeres: BDM1 (54.9\%), BDM2 (35.69\%) y BDM3 (9.41\%). Se observan diferencias significativas entre los 6 grupos, tanto en el número máximo de UBEs consumidas en una sesión de $\mathrm{BD}$, como en el número de veces que realizan $\mathrm{BD}$ en los últimos seis meses.

En las mujeres, cuanto mayor es la cantidad ingerida $(\mathrm{p}<.001)$, mayor es la frecuencia con la que realizan $\mathrm{BD}(\mathrm{p}<.001)$. En los varones, los grupos BDV2 y BDV3 realizan BD con mayor regularidad que el BDV1 $(\mathrm{p}<.001)$. En cantidad, destaca el grupo BDV3 sobre el BDV1 ( $\mathrm{p}<.001)$. Entre los grupos BDV2 y BDV3, el primero consume con mayor frecuencia en comparación con el segundo $(\mathrm{p}<.001)$, pero el segundo ingiere cantidades significativamente superiores al primero $(\mathrm{p}<.001)$.

Las comparaciones entre los seis grupos BDs, indican que los valores de los grupos de mujeres BDM2 y BDM3 son superiores a los de sus compañeros masculinos menos intensivos (BDV1), tanto en cantidad $(\mathrm{p}<.001)$ como en frecuencia $(\mathrm{p}<.001)$. Lo mismo ocurre entre los grupos de varones BDV2 y BDV3 respecto a las mujeres menos intensivas BDM1, donde también se aprecian diferencias en la cantidad $(\mathrm{p}<.001)$ y en la frecuencia $(\mathrm{p}<.001)$.

Por otra parte, al realizar comparaciones entre los estudiantes de ambos sexos que realizaban BD en menor cantidad y frecuencia -menos intensivos- (BDV1 y BDM1), éstos reali- 
zan consumos similares tanto en cantidad $(\mathrm{p}=.08)$ como en frecuencia $(\mathrm{p}=.751)$. Entre los chicos y chicas que consumen con mayor intensidad (BDV3 y BDM3) tampoco se observan diferencias en las cantidades ingeridas $(\mathrm{p}=.13)$, no siendo así en su frecuencia. Las mujeres BDM3 presentan un patrón más regular de BD que los varones BDV3 (p<.001). Cuando se atiende a estos dos grupos por separado, las mujeres BDM3 ingieren mayor cantidad de alcohol que los varones BDV2 ( $<<.001)$, mientras que los varones BDV3 realizan mayor ingesta que las mujeres BDM2 ( $\mathrm{p}<.001)$. Por su parte, los chicos del grupo BDV2 muestran un patrón de dicha conducta más frecuente que sus compañeras BDM2 (p<.001). En cambio, no muestran diferencias con respecto a las mujeres más intensivas (BDM3) (p=.775).

Tabla 1.- Comparación de medias ANOVA entre las variables cantidad y frecuencia de consumo, incluidas en los conglomerados BD.

$\mathrm{BD}(\mathrm{n}=384)$

\begin{tabular}{|c|c|c|c|c|c|c|c|c|}
\hline & \multicolumn{2}{|c|}{ Varones $(n=129)$} & \multicolumn{4}{|c|}{ Mujeres ( $n=255$ ) } & \multirow[b]{3}{*}{$F$} & \multirow[b]{3}{*}{$p$} \\
\hline & $\begin{array}{l}\text { BD1V } \\
(n=76)\end{array}$ & $\begin{array}{l}\text { BD2V } \\
(n=18)\end{array}$ & $\begin{array}{l}\text { BD3V } \\
(n=35)\end{array}$ & $\begin{array}{c}\text { BD1M } \\
(n=140)\end{array}$ & $\begin{array}{l}\text { BD2M } \\
(n=91)\end{array}$ & $\begin{array}{l}\text { BD3M } \\
(n=24)\end{array}$ & & \\
\hline & $\mathrm{M}(\mathrm{DT})$ & $M(D T)$ & $M(D T)$ & $M(D T)$ & $M(D T)$ & $\mathrm{M}(\mathrm{DT})$ & & \\
\hline UBES max/BD & $\begin{array}{c}11.63 \\
(3.436)\end{array}$ & $\begin{array}{c}15.72 \\
(5.367)\end{array}$ & $\begin{array}{c}29.09 \\
(8.143)\end{array}$ & $\begin{array}{c}9.21 \\
(2.614)\end{array}$ & $\begin{array}{c}17.85 \\
(5.953)\end{array}$ & $\begin{array}{c}24.92 \\
(17.847)\end{array}$ & 78,137 & .000 \\
\hline Frec BD/6m & $\begin{array}{c}4.36 \\
(3.212)\end{array}$ & $\begin{array}{c}27.94 \\
(10.298)\end{array}$ & $\begin{array}{c}10.51 \\
(5.741)\end{array}$ & $\begin{array}{c}3.45 \\
(2.352)\end{array}$ & $\begin{array}{c}10.54 \\
(4.542)\end{array}$ & $\begin{array}{c}26.13 \\
(9.284)\end{array}$ & 302,904 & .000 \\
\hline
\end{tabular}

Nota: BD1V= Grupo 1 de binge drinkers varones (BDs); BD2V= Grupo 2 de BDs varones; $\mathrm{BD} 3 \mathrm{~V}=$ Grupo 3 de BDs varones; $\mathrm{BD} 1 \mathrm{M}=$ Grupo 1 de BDs mujeres; BD2M= Grupo 2 de BDs mujeres; BD3M= Grupo 3 de BDs mujeres.

\section{Personalidad}

En la tabla 2 se muestran los resultados de las 5 dimensiones de personalidad y sus facetas. Entre las dimensiones, la Extraversión es la única que no muestra diferencias significativas entre los grupos. El grupo BDV2 muestra puntuaciones más bajas en Amabilidad en comparación con cualquier grupo de mujeres ( $\mathrm{p}<.027)$ y menor puntuación en Apertura en comparación con las NoBDM (p<.048). En Neuroticismo, son las chicas BDs menos intensivas (BDM1) y las más intensivas (BDM3) las que manifiestan niveles superiores con respecto a los grupos BDV1, BDV2 y NoBDV (p<.048). En relación con la Responsabilidad, son las NoBDM quienes muestran más puntuación que los chicos y chicas de los grupos BDM2, BDV2, BDM3 y BDV3 (p<.05).

Cuando se atiende a las facetas de la dimensión de Neuroticismo, las mujeres presentan siempre un mayor nivel de Ansiedad que los varones ( $<$.008). En la faceta Depresión son los varones que practican BD con mayor frecuencia (BDV2) los que puntúan más bajo que 
las mujeres BD menos intensivas (BDM1) y más intensivas (BDM3) (p<.048). Respecto a la Vulnerabilidad, las mujeres menos intensivas (BDM1) y moderadas (BDM2) puntúan más elevado en comparación con todos los grupos de varones $(\mathrm{p}<.022)$. Por último, las mujeres más intensivas (BDM3) muestran mayor Impulsividad respecto al resto de grupos $(\mathrm{p}<.029)$, exceptuando sus compañeros más intensivos (BDV3) $(\mathrm{p}=.312)$.

Aunque la dimensión de Extraversión no muestra diferencias significativas, sí lo hacen dos de sus facetas. Concretamente, el grupo de chicos BD que practica dicha conducta con mayor asiduidad (BDV2) muestra mayores niveles en Asertividad que las chicas BD menos intensivas (BDM1) (p<.047). Por su parte, los varones BD que ingiere cantidades mayores (BDV3) presentan una mayor Búsqueda de Sensaciones en comparación con el grupo de mujeres menos intensivas (BDM1) y las NoBDM (p<.006).

En relación con la dimensión de Apertura, todas las mujeres muestran mayores Sentimientos que los NoBDV ( $\mathrm{p}<.041)$. Respecto a la faceta de Valores todos los grupos de las mujeres BD presentan mayores niveles que los varones BD menos intensivos (BDV1) y los NoBDV ( $\mathrm{p}<.016)$. Por su parte, en la faceta Acciones, únicamente destaca el grupo de chicas BDM2 sobre los NoBDV (p<.005). En la última faceta de la dimensión Apertura, la denominada Estética, muestran mayores puntuaciones las mujeres BDM1 y BDM2, así como las NoBDM en comparación con los hombres que realizan BD con mayor frecuencia (BDV2) $(\mathrm{p}<.016)$.

En las facetas de la Amabilidad, el grupo BDV2 manifiesta menor Altruismo, Modestia y Sensibilidad a los Demás que cualquier grupo de mujeres $(\mathrm{p}<.011)$, y bajos niveles en Franqueza comparado con las NoBDM y las BD menos intensivas (BDM1) y moderadas (BDM2) $(\mathrm{p}<.028)$.

Por último, en Responsabilidad, las NoBDM muestran un mayor Sentido del Deber y Necesidad de Logro que el grupo BDV2 (p<.035). También, se observa que las estudiantes BD más intensivas (BDM3) presentan menor Autodisciplina que los estudiantes NoBDV y NoBDM (p<.039). Por último, en la faceta de Reflexión y Deliberación, las mujeres BD moderadas (BDM2) y más intensivas (BDM3), así como los chicos que realizan este patrón con mayor frecuencia (BDV2) y los que ingieren mayores cantidades (BDV3) manifiestan puntuaciones inferiores a las NoBDM $(\mathrm{p}<.025)$.

(Ver tabla 2)

\section{Discusión}

A diferencia de las aportaciones de investigaciones anteriores (Valencia-Martín, Galán y Rodríguez-Artalejo, 2007; Pilatti, Caneto, Garimaldi, Del Valle y Pautassi, 2013) en las que se confirmó un mayor nivel de consumo por parte de los varones universitarios BD, en el presente estudio se igualan los consumos entre sexos, independientemente del tipo de patrón de BD, tal como apuntaba Cortés et al. (2017a) con población adolescente. Este dato parece apoyar la tendencia observada al comparar diferentes estudios (Cortés et al., 2016; Cortés, Giménez, Motos, Sancerni y Cadaveira, 2017b) de que las adolescentes BD que consumían cantidades similares a las de sus compañeros masculinos están manteniendo 
Tabla 2.- Comparación de medias ANOVA entre las dimensiones y facetas de la personalidad del NEO-PI-R, según los grupos de los conglomerados BD y los grupos NoBD.

\begin{tabular}{|c|c|c|c|c|c|c|c|c|c|c|}
\hline & \multicolumn{6}{|c|}{$B D(n=384)$} & \multicolumn{2}{|c|}{ NoBD $(n=217)$} & \multirow[b]{4}{*}{$F$} & \multirow[b]{4}{*}{$p$} \\
\hline & \multicolumn{3}{|c|}{ Varones $(n=129)$} & \multicolumn{3}{|c|}{ Mujeres ( $n=255$ ) } & \multirow[b]{2}{*}{$\begin{array}{l}\text { NoBDM } \\
(n=130)\end{array}$} & \multirow[b]{2}{*}{$\begin{array}{c}\text { NoBDV } \\
(n=87)\end{array}$} & & \\
\hline & $\begin{array}{l}\text { BD1V } \\
(n=76)\end{array}$ & $\begin{array}{l}\text { BD2V } \\
(n=18)\end{array}$ & $\begin{array}{l}\text { BD3V } \\
(n=35)\end{array}$ & $\begin{array}{c}\text { BD1M } \\
(n=140)\end{array}$ & $\begin{array}{l}\text { BD2M } \\
(n=91)\end{array}$ & $\begin{array}{l}\text { BD3M } \\
(n=24)\end{array}$ & & & & \\
\hline & $M(D T)$ & $M(D T)$ & $M(D T)$ & $M(D T)$ & $M(D T)$ & $M(D T)$ & $M(D T)$ & $M(D T)$ & & \\
\hline Neuroticismo & $\begin{array}{c}85.96 \\
(18.050)\end{array}$ & $\begin{array}{c}79.82 \\
(20.604)\end{array}$ & $\begin{array}{c}90.86 \\
(21.119)\end{array}$ & $\begin{array}{c}96.64 \\
(19.307)\end{array}$ & $\begin{array}{c}93.97 \\
(15.640)\end{array}$ & $\begin{array}{c}100.25 \\
(21.436)\end{array}$ & $\begin{array}{c}94.21 \\
(21.682)\end{array}$ & $\begin{array}{c}86.68 \\
(18.224)\end{array}$ & 5.089 & .000 \\
\hline Ansiedad & $\begin{array}{c}15.85 \\
(4.076)\end{array}$ & $\begin{array}{c}13.24 \\
(4.790)\end{array}$ & $\begin{array}{c}17.00 \\
(4.845)\end{array}$ & $\begin{array}{c}19.19 \\
(4.493)\end{array}$ & $\begin{array}{c}18.46 \\
(4.438)\end{array}$ & $\begin{array}{c}19.08 \\
(5.307)\end{array}$ & $\begin{array}{c}19.14 \\
(4.599)\end{array}$ & $\begin{array}{c}16.52 \\
(4.570)\end{array}$ & 9.400 & .000 \\
\hline Hostilidad & $\begin{array}{c}13.17 \\
(3.685)\end{array}$ & $\begin{array}{c}14.44 \\
(5.762)\end{array}$ & $\begin{array}{c}14.06 \\
(4.576)\end{array}$ & $\begin{array}{c}14.82 \\
(3.943)\end{array}$ & $\begin{array}{c}14.51 \\
(3.758)\end{array}$ & $\begin{array}{c}15.00 \\
(5.389)\end{array}$ & $\begin{array}{c}14.57 \\
(5.291)\end{array}$ & $\begin{array}{c}13.38 \\
(4.138)\end{array}$ & 1.579 & .139 \\
\hline Depresión & $\begin{array}{c}13.41 \\
(5.269)\end{array}$ & $\begin{array}{c}10.50 \\
(4.048)\end{array}$ & $\begin{array}{c}14.49 \\
(5.002)\end{array}$ & $\begin{array}{c}14.66 \\
(5.749)\end{array}$ & $\begin{array}{c}13.87 \\
(4.918)\end{array}$ & $\begin{array}{c}16.50 \\
(6.379)\end{array}$ & $\begin{array}{c}14.26 \\
(5.888)\end{array}$ & $\begin{array}{c}13.30 \\
(4.989)\end{array}$ & 2.499 & .015 \\
\hline $\begin{array}{l}\text { Ansiedad } \\
\text { social }\end{array}$ & $\begin{array}{c}14.51 \\
(4.804)\end{array}$ & $\begin{array}{c}13.39 \\
(4.448)\end{array}$ & $\begin{array}{c}14.86 \\
(4.038)\end{array}$ & $\begin{array}{c}15.83 \\
(4.886)\end{array}$ & $\begin{array}{c}14.91 \\
(3.934)\end{array}$ & $\begin{array}{c}14.83 \\
(6.012)\end{array}$ & $\begin{array}{c}15.38 \\
(4.615)\end{array}$ & $\begin{array}{c}15.10 \\
(4.338)\end{array}$ & 1.124 & .346 \\
\hline Impulsividad & $\begin{array}{c}17.20 \\
(3.969)\end{array}$ & $\begin{array}{c}17.06 \\
(3.556)\end{array}$ & $\begin{array}{c}18.57 \\
(4.139)\end{array}$ & $\begin{array}{c}17.46 \\
(3.800)\end{array}$ & $\begin{array}{c}17.92 \\
(3.407)\end{array}$ & $\begin{array}{c}20.83 \\
(3.497)\end{array}$ & $\begin{array}{c}17.26 \\
(3.967)\end{array}$ & $\begin{array}{c}16.48 \\
(3.497)\end{array}$ & 4.354 & .000 \\
\hline Vulnerabilidad & $\begin{array}{l}11.95 \\
(4.511)\end{array}$ & $\begin{array}{c}11.82 \\
(5.053)\end{array}$ & $\begin{array}{c}11.89 \\
(4.770)\end{array}$ & $\begin{array}{c}14.53 \\
(4.308)\end{array}$ & $\begin{array}{c}14.11 \\
(3.582)\end{array}$ & $\begin{array}{c}14.00 \\
(4.511)\end{array}$ & $\begin{array}{c}13.61 \\
(4.120)\end{array}$ & $\begin{array}{c}11.90 \\
(3.918)\end{array}$ & 5.743 & .000 \\
\hline Extraversión & $\begin{array}{c}113.61 \\
(15.502)\end{array}$ & $\begin{array}{c}116.29 \\
(22.745)\end{array}$ & $\begin{array}{c}115.63 \\
(21.605)\end{array}$ & $\begin{array}{c}110.79 \\
(19.194)\end{array}$ & $\begin{array}{c}115.56 \\
(18.509)\end{array}$ & $\begin{array}{c}117.50 \\
(18.058)\end{array}$ & $\begin{array}{c}112.01 \\
(18.906)\end{array}$ & $\begin{array}{c}111.06 \\
(18.331)\end{array}$ & 1.080 & .374 \\
\hline $\begin{array}{c}\text { Cálido/ } \\
\text { Cordialidad }\end{array}$ & $\begin{array}{c}21.41 \\
(3.587)\end{array}$ & $\begin{array}{c}19.41 \\
(4.976)\end{array}$ & $\begin{array}{c}21.17 \\
(4.706)\end{array}$ & $\begin{array}{c}20.54 \\
(4.467)\end{array}$ & $\begin{array}{c}21.18 \\
(4.373)\end{array}$ & $\begin{array}{c}20.96 \\
(5.238)\end{array}$ & $\begin{array}{c}21.55 \\
(4.227)\end{array}$ & $\begin{array}{c}20.61 \\
(4.194)\end{array}$ & 1.098 & .363 \\
\hline Gregarismo & $\begin{array}{c}17.57 \\
(4.325)\end{array}$ & $\begin{array}{c}18.83 \\
(5.914)\end{array}$ & $\begin{array}{c}17.80 \\
(5.835)\end{array}$ & $\begin{array}{c}17.76 \\
(5.318)\end{array}$ & $\begin{array}{c}18.75 \\
(4.723)\end{array}$ & $\begin{array}{c}19.79 \\
(5.421)\end{array}$ & $\begin{array}{c}17.43 \\
(5.426)\end{array}$ & $\begin{array}{c}16.79 \\
(5.320)\end{array}$ & 1.630 & .124 \\
\hline Asertividad & $\begin{array}{c}17.32 \\
(4.192)\end{array}$ & $\begin{array}{c}20.11 \\
(3.909)\end{array}$ & $\begin{array}{c}18.06 \\
(4.359)\end{array}$ & $\begin{array}{c}16.59 \\
(4.889)\end{array}$ & $\begin{array}{c}17.04 \\
(4.637)\end{array}$ & $\begin{array}{c}16.92 \\
(5.149)\end{array}$ & $\begin{array}{c}16.71 \\
(4.310)\end{array}$ & $\begin{array}{c}17.97 \\
(4.826)\end{array}$ & 2.142 & .038 \\
\hline Actividad & $\begin{array}{c}18.11 \\
(3.711)\end{array}$ & $\begin{array}{c}20.06 \\
(4.007)\end{array}$ & $\begin{array}{c}18.57 \\
(4.320)\end{array}$ & $\begin{array}{c}18.05 \\
(4.318)\end{array}$ & $\begin{array}{c}18.44 \\
(4.290)\end{array}$ & $\begin{array}{c}20.21 \\
(3.978)\end{array}$ & $\begin{array}{c}18.28 \\
(4.076)\end{array}$ & $\begin{array}{c}17.86 \\
(4.090)\end{array}$ & 1.463 & .178 \\
\hline $\begin{array}{l}\text { Búsqueda } \\
\text { emociones }\end{array}$ & $\begin{array}{c}17.47 \\
(3.438)\end{array}$ & $\begin{array}{c}18.17 \\
(4.048)\end{array}$ & $\begin{array}{c}19.03 \\
(4.495)\end{array}$ & $\begin{array}{c}16.34 \\
(3.835)\end{array}$ & $\begin{array}{c}17.44 \\
(3.509)\end{array}$ & $\begin{array}{c}17.71 \\
(3.940)\end{array}$ & $\begin{array}{c}16.18 \\
(4.032)\end{array}$ & $\begin{array}{c}17.07 \\
(4.031)\end{array}$ & 3.448 & .001 \\
\hline $\begin{array}{c}\text { Emociones } \\
\text { positivas }\end{array}$ & $\begin{array}{c}21.73 \\
(4.542)\end{array}$ & $\begin{array}{c}20.00 \\
(5.568)\end{array}$ & $\begin{array}{l}21.00 \\
(6.311)\end{array}$ & $\begin{array}{c}21.69 \\
(4.556)\end{array}$ & $\begin{array}{c}22.73 \\
(4.328)\end{array}$ & $\begin{array}{c}21.92 \\
(5.090)\end{array}$ & $\begin{array}{c}21.87 \\
(5.307)\end{array}$ & $\begin{array}{c}20.76 \\
(4.256)\end{array}$ & 1.497 & .165 \\
\hline Apertura & $\begin{array}{c}109.99 \\
(19.124)\end{array}$ & $\begin{array}{c}103.65 \\
(14.026)\end{array}$ & $\begin{array}{c}112.31 \\
(20.423)\end{array}$ & $\begin{array}{c}114.93 \\
(15.818)\end{array}$ & $\begin{array}{c}115.89 \\
(16.555)\end{array}$ & $\begin{array}{c}114.78 \\
(21.030)\end{array}$ & $\begin{array}{c}116.29 \\
(19.031)\end{array}$ & $\begin{array}{c}107.49 \\
(18.628)\end{array}$ & 3.321 & .002 \\
\hline Fantasía & $\begin{array}{c}19.37 \\
(4.744)\end{array}$ & $\begin{array}{c}18.89 \\
(4.549)\end{array}$ & $\begin{array}{c}18.97 \\
(5.050)\end{array}$ & $\begin{array}{c}19.55 \\
(4.418)\end{array}$ & $\begin{array}{c}19.54 \\
(4.034)\end{array}$ & $\begin{array}{c}18.17 \\
(5.239)\end{array}$ & $\begin{array}{c}19.57 \\
(4.945)\end{array}$ & $\begin{array}{c}18.52 \\
(4.382)\end{array}$ & .778 & .606 \\
\hline Estética & $\begin{array}{c}16.75 \\
(5.512)\end{array}$ & $\begin{array}{c}13.89 \\
(3.179)\end{array}$ & $\begin{array}{c}16.83 \\
(5.928)\end{array}$ & $\begin{array}{c}19.04 \\
(5.054)\end{array}$ & $\begin{array}{c}18.53 \\
(4.900)\end{array}$ & $\begin{array}{c}16.52 \\
(5.799)\end{array}$ & $\begin{array}{c}19.82 \\
(5.285)\end{array}$ & $\begin{array}{c}16.13 \\
(5.630)\end{array}$ & 7.337 & .000 \\
\hline
\end{tabular}




\begin{tabular}{|c|c|c|c|c|c|c|c|c|c|c|}
\hline Sentimientos & $\begin{array}{c}20.35 \\
(3.896)\end{array}$ & $\begin{array}{c}19.56 \\
(3.792)\end{array}$ & $\begin{array}{c}20.20 \\
(4.028)\end{array}$ & $\begin{array}{c}20.99 \\
(3.559)\end{array}$ & $\begin{array}{l}21.33 \\
(3.325)\end{array}$ & $\begin{array}{c}22.21 \\
(5.183)\end{array}$ & $\begin{array}{l}21.15 \\
(3.729)\end{array}$ & $\begin{array}{c}19.39 \\
(3.677)\end{array}$ & 3.258 & .002 \\
\hline Acciones & $\begin{array}{c}15.43 \\
(3.728)\end{array}$ & $\begin{array}{c}15.22 \\
(3.282)\end{array}$ & $\begin{array}{c}16.71 \\
(3.268)\end{array}$ & $\begin{array}{c}16.23 \\
(2.996)\end{array}$ & $\begin{array}{c}17.01 \\
(3.526)\end{array}$ & $\begin{array}{c}16.79 \\
(3.912)\end{array}$ & $\begin{array}{l}16.57 \\
(3.829)\end{array}$ & $\begin{array}{l}15.06 \\
(3.535)\end{array}$ & 3.147 & .003 \\
\hline Ideas & $\begin{array}{c}18.91 \\
(5.926)\end{array}$ & $\begin{array}{c}18.11 \\
(4.378)\end{array}$ & $\begin{array}{c}19.40 \\
(6.279)\end{array}$ & $\begin{array}{c}17.99 \\
(5.001)\end{array}$ & $\begin{array}{c}17.99 \\
(5.384)\end{array}$ & $\begin{array}{c}19.00 \\
(7.211)\end{array}$ & $\begin{array}{c}18.63 \\
(5.410)\end{array}$ & $\begin{array}{c}19.15 \\
(5.895)\end{array}$ & .680 & .689 \\
\hline Valores & $\begin{array}{c}19.19 \\
(3.660)\end{array}$ & $\begin{array}{c}19.29 \\
(4.934)\end{array}$ & $\begin{array}{c}20.20 \\
(3.420)\end{array}$ & $\begin{array}{c}20.95 \\
(3.264)\end{array}$ & $\begin{array}{l}21.42 \\
(3.624)\end{array}$ & $\begin{array}{c}21.58 \\
(2.873)\end{array}$ & $\begin{array}{c}20.63 \\
(3.459)\end{array}$ & $\begin{array}{l}19.25 \\
(4.155)\end{array}$ & 4.654 & .000 \\
\hline Amabilidad & $\begin{array}{c}109.44 \\
(17.971)\end{array}$ & $\begin{array}{c}96.63 \\
(17.424)\end{array}$ & $\begin{array}{c}108.83 \\
(20.351)\end{array}$ & $\begin{array}{c}114.48 \\
(16.243)\end{array}$ & $\begin{array}{c}116.04 \\
(15.914)\end{array}$ & $\begin{array}{l}114.88 \\
(21.138)\end{array}$ & $\begin{array}{c}117.28 \\
(17.166)\end{array}$ & $\begin{array}{c}108.55 \\
(18.163)\end{array}$ & 5.211 & .000 \\
\hline Confianza & $\begin{array}{c}17.56 \\
(3.710)\end{array}$ & $\begin{array}{c}16.67 \\
(4.765)\end{array}$ & $\begin{array}{c}18.23 \\
(5.694)\end{array}$ & $\begin{array}{c}17.26 \\
(4.264)\end{array}$ & $\begin{array}{l}17.67 \\
(3.844)\end{array}$ & $\begin{array}{c}17.50 \\
(4.433)\end{array}$ & $\begin{array}{c}18.31 \\
(4.195)\end{array}$ & $\begin{array}{c}17.34 \\
(4.046)\end{array}$ & 913 & .496 \\
\hline $\begin{array}{l}\text { Honradezl } \\
\text { Franqueza }\end{array}$ & $\begin{array}{c}16.27 \\
(5.084)\end{array}$ & $\begin{array}{c}15.06 \\
(3.208)\end{array}$ & $\begin{array}{c}16.49 \\
(4.623)\end{array}$ & $\begin{array}{c}18.44 \\
(4.272)\end{array}$ & $\begin{array}{c}18.67 \\
(4.372)\end{array}$ & $\begin{array}{c}17.92 \\
(5.445)\end{array}$ & $\begin{array}{c}18.76 \\
(4.442)\end{array}$ & $\begin{array}{c}17.31 \\
(4.823)\end{array}$ & 4.260 & .000 \\
\hline Altruismo & $\begin{array}{c}21.35 \\
(4.042)\end{array}$ & $\begin{array}{c}18.22 \\
(4.583)\end{array}$ & $\begin{array}{c}21.29 \\
(5.182)\end{array}$ & $\begin{array}{c}21.78 \\
(3.817)\end{array}$ & $\begin{array}{l}22.14 \\
(3.891)\end{array}$ & $\begin{array}{c}21.88 \\
(4.397)\end{array}$ & $\begin{array}{c}22.54 \\
(3.860)\end{array}$ & $\begin{array}{c}20.80 \\
(4.023)\end{array}$ & 3.599 & .001 \\
\hline $\begin{array}{l}\text { Actitud conci- } \\
\text { liadora }\end{array}$ & $\begin{array}{c}15.84 \\
(4.120)\end{array}$ & $\begin{array}{c}13.88 \\
(4.961)\end{array}$ & $\begin{array}{c}14.20 \\
(4.999)\end{array}$ & $\begin{array}{c}15.46 \\
(4.376)\end{array}$ & $\begin{array}{c}15.49 \\
(3.758)\end{array}$ & $\begin{array}{c}14.08 \\
(3.977)\end{array}$ & $\begin{array}{c}15.89 \\
(4.237)\end{array}$ & $\begin{array}{c}15.20 \\
(4.209)\end{array}$ & 1.437 & .188 \\
\hline Modestia & $\begin{array}{c}17.93 \\
(4.533)\end{array}$ & $\begin{array}{c}15.00 \\
(3.481)\end{array}$ & $\begin{array}{c}17.57 \\
(4.754)\end{array}$ & $\begin{array}{c}19.44 \\
(4.146)\end{array}$ & $\begin{array}{c}19.60 \\
(4.005)\end{array}$ & $\begin{array}{c}20.42 \\
(6.199)\end{array}$ & $\begin{array}{c}19.07 \\
(4.342)\end{array}$ & $\begin{array}{c}17.25 \\
(5.014)\end{array}$ & 5.393 & .000 \\
\hline $\begin{array}{l}\text { Sensibilidad a } \\
\text { los demás }\end{array}$ & $\begin{array}{c}20.49 \\
(3.853)\end{array}$ & $\begin{array}{c}18.71 \\
(4.985)\end{array}$ & $\begin{array}{c}21.06 \\
(4.087)\end{array}$ & $\begin{array}{c}22.23 \\
(3.849)\end{array}$ & $\begin{array}{c}22.55 \\
(3.928)\end{array}$ & $\begin{array}{c}23.08 \\
(4.333)\end{array}$ & $\begin{array}{c}22.92 \\
(3.620)\end{array}$ & $\begin{array}{c}20.64 \\
(3.828)\end{array}$ & 6.647 & .000 \\
\hline $\begin{array}{l}\text { Responsabi- } \\
\quad \text { lidad }\end{array}$ & $\begin{array}{l}107.68 \\
(21.155)\end{array}$ & $\begin{array}{c}101.71 \\
(20.515)\end{array}$ & $\begin{array}{c}103.54 \\
(19.521)\end{array}$ & $\begin{array}{c}112.34 \\
(18.409)\end{array}$ & $\begin{array}{c}107.03 \\
(18.980)\end{array}$ & $\begin{array}{l}103.75 \\
(23.804)\end{array}$ & $\begin{array}{c}116.11 \\
(19.616)\end{array}$ & $\begin{array}{c}112.80 \\
(19.408)\end{array}$ & 3.935 & .000 \\
\hline Competencia & $\begin{array}{c}19.56 \\
(3.946)\end{array}$ & $\begin{array}{l}20.06 \\
(4.108)\end{array}$ & $\begin{array}{c}19.46 \\
(4.822)\end{array}$ & $\begin{array}{c}20.14 \\
(3.667)\end{array}$ & $\begin{array}{c}19.41 \\
(3.927)\end{array}$ & $\begin{array}{c}18.83 \\
(5.577)\end{array}$ & $\begin{array}{c}20.44 \\
(3.991)\end{array}$ & $\begin{array}{c}20.28 \\
(3.839)\end{array}$ & 1.132 & .341 \\
\hline Orden & $\begin{array}{c}17.13 \\
(4.869)\end{array}$ & $\begin{array}{c}14.83 \\
(5.272)\end{array}$ & $\begin{array}{c}16.00 \\
(4.684)\end{array}$ & $\begin{array}{c}17.74 \\
(4.700)\end{array}$ & $\begin{array}{l}16.95 \\
(5.263)\end{array}$ & $\begin{array}{c}15.71 \\
(5.188)\end{array}$ & $\begin{array}{c}18.34 \\
(4.709)\end{array}$ & $\begin{array}{c}17.20 \\
(4.623)\end{array}$ & 2.051 & .052 \\
\hline $\begin{array}{l}\text { Sentido del } \\
\text { deber }\end{array}$ & $\begin{array}{c}18.53 \\
(3.957)\end{array}$ & $\begin{array}{c}18.22 \\
(4.772)\end{array}$ & $\begin{array}{c}18.37 \\
(3.482)\end{array}$ & $\begin{array}{c}19.51 \\
(3.425)\end{array}$ & $\begin{array}{c}19.08 \\
(3.177)\end{array}$ & $\begin{array}{c}19.50 \\
(3.730)\end{array}$ & $\begin{array}{c}20.31 \\
(3.098)\end{array}$ & $\begin{array}{l}19.36 \\
(3.804)\end{array}$ & 2.702 & .009 \\
\hline $\begin{array}{l}\text { Necesidad del } \\
\text { deber }\end{array}$ & $\begin{array}{c}18.43 \\
(4.536)\end{array}$ & $\begin{array}{c}17.83 \\
(3.959)\end{array}$ & $\begin{array}{c}18.60 \\
(4.313)\end{array}$ & $\begin{array}{c}19.53 \\
(4.097)\end{array}$ & $\begin{array}{c}19.23 \\
(4.214)\end{array}$ & $\begin{array}{c}19.08 \\
(5.778)\end{array}$ & $\begin{array}{c}20.46 \\
(4.357)\end{array}$ & $\begin{array}{c}19.86 \\
(4.688)\end{array}$ & 2.231 & .030 \\
\hline Autodisciplina & $\begin{array}{c}17.53 \\
(4.515)\end{array}$ & $\begin{array}{c}16.82 \\
(4.111)\end{array}$ & $\begin{array}{c}16.60 \\
(3.897)\end{array}$ & $\begin{array}{c}17.78 \\
(4.277)\end{array}$ & $\begin{array}{c}16.87 \\
(3.859)\end{array}$ & $\begin{array}{c}15.96 \\
(4.506)\end{array}$ & $\begin{array}{c}18.58 \\
(4.109)\end{array}$ & $\begin{array}{c}18.66 \\
(4.371)\end{array}$ & 2.989 & .004 \\
\hline $\begin{array}{l}\text { Reflexión y } \\
\text { deliberación }\end{array}$ & $\begin{array}{c}16.49 \\
(4.825)\end{array}$ & $\begin{array}{c}14.18 \\
(5.126)\end{array}$ & $\begin{array}{c}14.51 \\
(4.859)\end{array}$ & $\begin{array}{c}17.55 \\
(4.850)\end{array}$ & $\begin{array}{c}15.67 \\
(4.860)\end{array}$ & $\begin{array}{c}14.67 \\
(4.508)\end{array}$ & $\begin{array}{c}18.15 \\
(4.763)\end{array}$ & $\begin{array}{c}17.25 \\
(3.980)\end{array}$ & 5.542 & .000 \\
\hline
\end{tabular}

Nota: $\mathrm{BD} 1 \mathrm{~V}=$ Grupo 1 de binge drinkers varones (BDs); BD2V= Grupo 2 de BDs varones; $\mathrm{BD} 3 \mathrm{~V}=$ Grupo 3 de BDs varones; BD1M= Grupo 1 de BDs mujeres; BD2M= Grupo 2 de BDs mujeres; BD3M= Grupo 3 de BDs mujeres; NoBDM= Grupo de mujeres No BDs; NoBDV= Grupo de varones No BDs. 
este nivel de ingesta tras alcanzar la mayoría de edad, incluso superando la frecuencia de realización de esta ingesta, tal como se muestra en el presente estudio.

Es importante atender, en los próximos años, a la evolución de esta tendencia en el consumo por parte de las mujeres, dado que éstas son más vulnerables a las consecuencias de la ingesta de alcohol (Wechsler y Nelson, 2010), incrementándose notablemente el riesgo asociado a este patrón de consumo.

Además, se confirma que prácticamente casi la mitad de estos jóvenes, independientemente del sexo, al menos duplican los niveles de BD, con una regularidad de una vez por semana, constituyendo poblaciones de alto riesgo (Cortés et al. 2017a; Cortés et al., 2017b; Parada et al., 2011; Livingston, 2013).

Estos resultados obligan a reflexionar sobre la necesidad de implementar medidas preventivas efectivas en esta población de cara a reducir las consecuencias biopsicosociales experimentadas por estos colectivos a medio y largo plazo (ESPAD, 2016; Parada et al., 2011).

Además, destaca la necesidad de replantearse la utilidad de evaluar el BD a partir de un único punto de corte. Esta clasificación dicotómica enmascara la heterogeneidad de consumidores BD que existen y por tanto es insuficiente para caracterizar adecuadamente los hábitos de consumo e intervenir sobre las consecuencias reales asociadas a las particularidades de esta conducta (Cortés, Motos y Giménez, 2013; Patrick et al., 2013).

Cuando se atiende a la personalidad es importante tener presente en varones que existe cierta relación entre un mayor número de rasgos de personalidad y una elevada frecuencia de realización del $\mathrm{BD}$.

Estos varones se caracterizan por ser muy sociables y dominantes, lo que les expone a situaciones en las que habitualmente se consume alcohol. También, presentan bajos niveles en la mayoría de las facetas de Amabilidad y Responsabilidad, lo que los define como individualistas y egocéntricos, con dificultades para seguir las normas sociales y con poco interés en objetivos en la vida. Además, presentan menor preocupación social, suelen ser negligentes con su propio bienestar y con el de los que le rodean, mostrando una mayor facilidad para realizar comportamientos peligrosos tanto para ellos como para los demás, sin importarles las consecuencias.

Conocer estas particularidades permite guiar la intervención hacia actividades que trabajen los valores prosociales (sentido del deber, respecto, solidaridad, coorperación, etc), y que fomenten la empatía, la motivación de logro y las habilidades de comunicación basadas en la escucha activa y la asertividad. Todo ello les permitirá disponer de herramientas para saber gestionar de manera adecuada situaciones sociales de consumo, incrementar su preocupación por el bienestar de los demás, mantener unos principios éticos y morales y fomentar su interés por trabajar en lograr sus objetivos.

A diferencia de lo esperable (Adan et al., 2017; Erevik et al., 2017; Hakulinen y Jokela, 2018; Lac y Donaldson, 2016; Lang et al., 2012) sólo los chicos que ingieren mayores cantidades de alcohol muestran una fuerte necesidad por buscar nuevas experiencias, siendo más desinhibidos y mostrando mayor susceptibilidad al aburrimiento. 
En estos casos, un recurso útil puede ser la práctica de actividades deportivas, ayudando a que los jóvenes experimenten nuevas sensaciones y experiencias de manera saludable. En la revisión realizada por Barbero y Albéniz (2008) en adolescentes, jóvenes y adultos buscadores de sensaciones se concluyó que el realizar deporte es una de las herramientas más utilizadas para transmitir una serie de valores y actitudes basadas en la confianza, responsabilidad, autocontrol y cooperación con los demás, transfiriendo lo aprendido a otros ámbitos de la vida donde el consumo puede estar presente.

En relación con el Neuroticismo, coincidiendo con los estudios previos (Ibáñez et al. 2010; Kuntsche, Kuntsche, Thrul y Gmel, 2017), las mujeres BD son las que muestran una mayor puntuación en esta dimensión, así como en algunas de sus facetas como Ansiedad, Depresión y Vulnerabilidad. Estos resultados confirman la necesidad de intervenir sobre estos rasgos para frenar la presencia de sentimientos negativos (miedo, melancolía, vergüenza, culpabilidad, etc.), ideas irracionales y dificultades para afrontar situaciones de estrés (Adan et al., 2017; Lac y Donaldson, 2016). En este sentido, la literatura científica (Kun y Demotrovics, 2010) considera que la Inteligencia Emocional actúa como variable mediadora entre los acontecimientos de la vida y las consecuencias sobre la salud y el bienestar, como la ansiedad y la depresión, ya que niveles elevados en esta habilidad emocional facilita la adaptabilidad de las personas a múltiples sucesos vitales, deteniendo los estados emocionales negativos y prolongando los positivos, de esta manera se favorece una respuesta adaptativa, por lo que es relevante poner en marcha actuaciones preventivas que fomenten la Inteligencia Emocional.

Un dato destacable entre las mujeres que realizan un BD con mayor frecuencia y cantidad de alcohol (más intensivas) es el alto nivel de Impulsividad mostrado, rasgo habitualmente característico entre la población masculina BD (Adan et al., 2017; Lang et al., 2012). Parece ser que las chicas que realizan un BD más extremo muestran problemas para controlar sus impulsos, aunque esto les lleve más adelante a lamentarse de dichos comportamientos. La presencia de las facetas de Neuroticismo mencionadas anteriormente puede llevarles a realizar consumos de riesgo por la creencia de que el alcohol alivia la angustia y la tristeza a corto plazo, aunque las aumenta a largo plazo. Esto puede conducir a un círculo vicioso repitiéndose la conducta en mayores ocasiones (Erevik et al., 2017) sin tener control sobre ello, lo que comporta un grave peligro en la aparición de trastornos por consumo de alcohol en un futuro. En esta situación sería relevante realizar intervenciones focalizadas en el establecimiento de límites o autocontrol en las respuestas impulsivas.

Es importante destacar que a diferencia de las conclusiones aportadas por los trabajos anteriores sobre la importancia de la dimensión de Responsabilidad entre los BD (Winogra, Steinley y Sher, 2014; Ibáñez et al., 2010; Mezquita et al., 2010; Ruiz et al., 2003), en este estudio solamente los chicos y chicas que realizan un BD moderado e intensivo han mostrado una baja Responsabilidad, así como en algunas de las facetas. Estas particularidades se han podido detectar dado que en este estudio, a diferencia de los anteriores, se ha tenido en cuenta los diferentes tipos de consumidores BD, según la cantidad y la frecuencia de ingesta de alcohol. De esta manera se ha obtenido información más detallada sobre el comportamiento de esta dimensión de personalidad en función del nivel de gravedad de este patrón de consumo. 
Concretamente, los que han puntuado bajo en esta dimensión se caracterizan por hablar y actuar de manera precipitada, sin tener en cuenta las consecuencias, y son incapaces de analizar las diferentes opciones antes de dar una respuesta. Además, las chicas más intensivas presentan una baja capacidad de motivación en tareas rutinarias. En este sentido, sería recomendable que la intervención se encaminase a desarrollar habilidades de resolución de conflictos, con el fin de aumentar la capacidad para solucionar problemas, analizando previamente la situación. Para ello, sería importante entrenar la escucha activa y la comprensión de los motivos que defienden todas las partes con el fin de buscar alternativas beneficiosas para todos. También, es relevante fortalecer la motivación de logro entre las mujeres más consumidoras. A diferencia de las conclusiones aportadas por Martin et al. (2015) en las que las mujeres BD presentaban una elevada Apertura, en este estudio sólo se observa la presencia de esta dimensión entre las chicas NoBD. No obstante, cuando se atiende a las facetas de orden inferior, las mujeres BD, sobre todo las que realizan un consumo moderado, destacan en algunas de ellas. Son personas que les dan importancia a los sentimientos y emociones, tolerantes hacia los nuevos cambios y con cierta disposición para cuestionarse valores sociales, políticos y religiosos. Asimismo, las mujeres, independientemente de su consumo presentan una elevada Amabilidad, así como en algunas de sus facetas, que las hace ser personas muy sinceras, humildes y preocupadas por el bienestar de los demás. Ambas dimensiones de personalidad actúan como factores de protección ante el consumo (Erevik, et al., 2017).

Dadas las puntaciones poco relevantes en los varones en estas dimensiones sería recomendable promover acciones que intervengan sobre el pensamiento crítico, y promuevan el altruismo y la movilización social. Estas medidas favorecen que las personas desarrollen capacidad para tomar decisiones por ellas mismas, espíritu crítico e interés por la seguridad de los demás.

A partir de las conclusiones aportadas sobre el papel de la personalidad entre los diferentes tipos de $\mathrm{BD}$, se advierte que desarrollar investigaciones, como las realizadas hasta ahora (Erevik et al., 2017; Hakulinen y Jokela, 2018; Lac y Donaldson, 2016), que no atienden a la gravedad del $\mathrm{BD}$, enmascara la existencia de ciertas relaciones entre algunas facetas de personalidad y los consumidores $\mathrm{BD}$, lo que dificulta enormemente poder ofrecer una intervención ajustada a sus necesidades.

Una de las limitaciones a considerar es el uso de autoinformes para determinar el patrón de consumo. Sin embargo, en la población juvenil, los autoinformes se han considerado válidos y fiables, ya que aseguran el anonimato de los datos, a diferencia de lo que suele ocurrir con otros tipos de registros, como las encuestas enviadas a hogares (Degenhardt et al., 2013).

Otra limitación radica en la generalización de los resultados obtenidos en este estudio, ya que este consumo está bastante presente en población joven más adulta. Sería interesante evaluar el papel de la personalidad en jóvenes entre los 20 y 29 años, periodo de mayor prevalencia del BD en España (OEDA, 2017). 
Además, en futuras investigaciones, sería interesante incorporar otras variables explicativas del BD, como las actitudes, motivos, expectativas o metacogniciones del consumo, ya que se ha comprobado que actúan como variables mediadoras en las relaciones entre personalidad y BD (De Wever y Quaglino, 2017), así como en la predicción de problemas relacionados con el alcohol (Lang et al., 2012; Mushquash et al., 2014).

\section{Referencias}

Adan, A., Forero, D. A., y Navarro, J. F. (2017). Personality traits related to binge drinking: a systematic review. Frontiers in psychiatry, 8, 134. doi: 10.3389/fpsyt.2017.00134

Barbero, V. R., y de Albéniz, G. G. P. (2008). La variable de personalidad búsqueda de sensaciones como factor de riesgo en el consumo de drogas. International Journal of Developmental and Educational Psychology, 4 , $169-178$.

Bravo, A. J., Pearson, M. R., Pilatti, A., Read, J. P., Mezquita, L., Ibáñez, M. I., y Ortet, G. (2018). Impulsivity-related traits, college alcohol beliefs, y alcohol outcomes: Examination of a prospective multiple mediation model among college students in Spain, Argentina, y USA. Addictive behaviors, 81, 125-133. doi: 10.1016/j.addbeh.2018.02.009

Cortés, M. T., Giménez, J. A., Motos, P., y Sancerni, M. D. (2016). Different versions of the Alcohol Use Disorders Identification Test (AUDIT) as screening instruments for underage binge drinking. Drug and Alcohol Dependence, 158, 52-59. doi:10.1016/j.drugalcdep.2015.10.033.

Cortés, M. T., Giménez, J. A., Motos, P., y Sancerni, M. D. (2017a). Revision of AUDIT consumption items to improve the screening of youth Binge Drinking. Frontiers in Psychology, 8, 910. doi:10.1016/j.drugalcdep.2015.10.033.

Cortés, M. T., Giménez, J. A., Motos, P., Sancerni, M. D., y Cadaveira, F. (2017b). The utility of the Alcohol Use Disorders Identification Test (AUDIT) for the analysis of binge drinking in university students. Psicothema, 29, 2. doi: 10.7334/psicothema2016.271.

Cortés, M. T., Giménez, J. A., Tomás, I., Espejo, B., Pascual, F., Pedrero, E., y Guardia, J. (2012). Instrumento de Evaluación del Consumo Intensivo de Alcohol. Informe final proyecto de investigación presentado al Plan Nacional sobre Drogas.

Cortés, M. T. y Motos, P. (2015). Cómo definir y medir el Consumo Intensivo de Alcohol. In M. T. Cortés (Ed.). Guía Clínica. Consumo intensivo de alcohol en jóvenes (pp. 25-46). Barcelona, España: Socidrogalcohol.

Cortés, M. T., Motos, P. y Giménez, J. A. (2013). Adjustment of different ways of measuring alcohol binge drinking in Spanish youngsters. Poster presentado en el Congreso Internacional Global Addiction, Pisa.

McCrae, R. R., y Costa, P. T. (1999). A five-factor theory of personality. Handbook of personality: Theory and research, 2, 139-153.

De Wever, E., y Quaglino, V. (2017). Risk factors and psychological vulnerabilities to binge-drinking in youth. L'Encephale, 43, 486-490. doi:10.1016/j.encep.2016.11.004

Degenhardt, L., O'Loughlin, C., Swift, W., Romaniuk, J. C., Coffey, C., Hall, W., y Patton, G. (2013). The persistence of adolescent binge drinking into adulthood: findings from a 15-year prospective cohort study. BMJ Open, 3, 1-11. doi: 10.1136/bmjopen-2013-003015.

Erevik, E. K., Pallesen, S., Vedaa, Ø., Yreassen, C. S., y Torsheim, T. (2017). Alcohol use among Norwegian students: Demographics, personality y psychological health correlates of drinking patterns. Nordic Studies on Alcohol y Drugs, 34, 415-429. doi: 10.1177/1455072517709918

ESPAD Group (2016), ESPAD Report 2015: Results from the European School Survey Project on Alcohol y Other Drugs, Publications Office of the European Union, Luxembourg. 
Hakulinen, C., y Jokela, M. (2018). Alcohol use y personality trait change: pooled analysis of six cohort studies. Psychological medicine, 1-8. doi: 10.1017/S0033291718001636

Ibanez, M. I., Camacho, L., Mezquita, L., Villa, H., Moya-Higueras, J., y Ortet, G. (2015). Alcohol expectancies mediate y moderate the associations between big five personality traits y adolescent alcohol consumption y alcoholrelated problems. Frontiers in Psychology, 6, 1838. doi:10.3389/fpsyg.2015.01838

Ibáñez, M. I., Moya, J., Villa, H., Mezquita, L., Ruipérez, M. Á., y Ortet, G. (2010). Basic personality dimensions y alcohol consumption in young adults. Personality y Individual Differences, 48, 171-176. doi: 10.1016/j. paid.2009.09.017

Kun, B., y Demetrovics, Z. (2010). Emotional intelligence and addictions: a systematic review. Substance use and misuse, 45, 1131-1160. doi: 10.3109/10826080903567855

Kuntsche, E., Kuntsche, S., Thrul, J., y Gmel, G. (2017). Binge drinking: health impact, prevalence, correlates and interventions. Psychology y health, 32,976-1017. doi: https://doi.org/10.1080/08870446.2017.1325889

Lac, A., y Donaldson, C. D. (2016). Alcohol attitudes, motives, norms, y personality traits longitudinally classified nondrinkers, moderate drinkers, y binge drinkers using discriminant functions analysis. Addictive Behaviors, 61, 91-8. doi:10.1016/j.addbeh.2016.05.006

Lang, K., Murphy, J. G., Monahan, C. J., Dennhardt, A. A., Skidmore, J. R., y McDevitt-Murphy, M. E. (2012). The role of positive consequences of alcohol in the relation between sensation seeking and drinking. Addiction Research \& Theory, 20, 504-510. doi:10.3109/16066369.2012.667854

Lannoy, S., Billieux, J., Poncin, M., y Maurage, P. (2017). Binging at the campus: motivations and impulsivity influence binge drinking profiles in university students. Psychiatry research, 250, 146-154. doi:10.1016/j.psychres.2017.01.068

Livingston, M. (2013). To reduce alcohol related harm we need to look beyond pubs and night clubs. Drug and A/cohol Review, 32, 113-114. doi: 10.1111/dar.12026

Marczinski, C. A., Grant, E. C., y Grant, V. J. (2009). Binge drinking in adolescents y college students. Nueva York: Nova Science.

Martin, J. L., Groth, G., Longo, L., Rocha, T. L., y Martens, M. P. (2015). Disordered eating and alcohol use among college women: associations with race and big five traits. Eating behaviors, 17, 149-152. doi:10.1016/j.eatbeh.2015.02.002

Mezquita, L., Stewart, H. S., y Ruipérez, M. A. (2010). Big five personality domains predict internal drinking motives in Young adults. Personality and Individual Differences, 49, 240-245. doi: 10.1016/j.paid.2010.03.043

Motos, P., Cortés, M. T., Giménez, J. A., y Cadaveira, F. (2015). Predictors of weekly alcohol drinking and alcohol-related problems in binge-drinking undergraduates. Adicciones, 27, 119-131.

Mushquash, C. J., Stewart, S. H., Mushquash, A. R., Comeau, M. N., y McGrath, P. J. (2014). Personality traits y drinking motives predict alcohol misuse among Canadian aboriginal youth. International Journal of Mental Health and Addiction, 12, 270-282. doi:10.1007/s11469-013-9451-4

Observatorio Español sobre Drogas y las Adicciones [OEDA] (2017). Informe 2017: EDADES, Encuesta sobre Alcohol y Drogas en España. Delegación del Gobierno para el Plan Nacional sobre Drogas. Madrid, España: Ministerio de Sanidad y Política Social.

Parada, M., Corral, M., Caamaño-Isorna, F., Mota, N., Crego, A., Rodríguez Holguín, S. y Cadaveira, F. (2011). Definition of adolescent binge drinking. Adicciones, 23, 53-63. doi:10.1016/j.alcohol.2011.10.002

Patrick, M. E., Schulenberg, J. E., Martz, M. E., Maggs, J. L., O’Malley, P. M., y Johnston, L. D. (2013). Extreme binge drinking among 12th-grade students in the United States: prevalence and predictors. JAMA pediatrics, 167, 1019-1025. doi:10.1001/jamapediatrics.2013.2392.

Pilatti, A., Caneto, F., Garimaldi, J. A., Del Valle, B., y Pautassi, R. M. (2013). Contribution of Time of Drinking Onset and Family History of Alcohol Problems in Alcohol and Drug Use Behaviors in Argentinean College Students. Alcohol and Alcoholism, 49, 128-137, doi:10.1093/alcalc/agt176.

Rodríguez-Martos, A., Gual Solé, A., y Llopis Llácer, J. J. (1999). The "standard drink unit" as a simplified recording system of alcohol consumption and its measurement in Spain. Medicina Clínica, 112, 446-450. 
Ruiz, M. A., Pincus, A. L., y Dickinson, K. A. (2003). NEO PI-R predictors of alcohol use y alcohol-related problems. Journal of personality assessment, 81,226-236. doi: 10.1207/S15327752JPA8103_05

Substance Abuse y Mental Health Services Administration. (2009). Results from the 2008 national survey on drug use y health: Summary of national findings. NSDUH Series H-36, HHS Publication No. SMA 09-4434. Rockville, MD: Substance Abuse y Mental Health Services Administration.

Valencia Martín, J. L., Galán, I., y Rodríguez Artalejo, F. (2007). Binge drinking in Madrid, Spain. Alcoholism: Clinical and Experimental Research, 31, 1723-1730. doi: 10.1111/j.1530-0277.2007.00473.x

Wechsler, H., y Nelson, T. F. (2010). Will increasing alcohol availability by lowering the minimum legal drinking age decrease drinking y related consequences among youths? American journal of public health, 100,986992. doi: 10.2105/AJPH.2009.178004

Winograd, R. P., Steinley, D. L., y Sher, K. J. (2014). Drunk personality: Reports from drinkers and knowledgeable informants. Experimental and clinical psychopharmacology, 22, 187. doi:10.1037/a0036607

Zhang, J., Bray, B. C., Zhang, M., y Lanza, S. T. (2015). Personality profiles and frequent heavy drinking in young adulthood. Personality and individual differences, 80, 18-21. doi:10.1016/j. paid.2015.01.054 